\title{
Comparison of Multilevel Inverters Employing DC Voltage Sources Scaled in the Power of Three
}

\author{
Seok-Hwan Hyun*, Cheol-soon Kwon*, kwang-soo Kim* and Feel-soon Kang*
}

\begin{abstract}
Cascaded H-bridge multilevel inverters shows a useful circuit configuration to increase the number of output voltage levels to obtain high quality output voltage. By applying the concept of the power of three to de voltage sources, it can increase the number of output voltage levels effectively. To realize this concept, two approaches may be considered. One is to use independent dc voltage sources pre-scaled in the power of three, and the other is to use instantaneous de voltage sources generated from a cascaded transformer, which has the secondary turn-ratios scaled in the power of three in sequence. A common feature in both approaches is to use the concept of the power of three for dc voltage sources, and a point of difference is whether it adopts a low frequency transformer or not, and where the transformer is located. According to the difference, application areas are limited and show different characteristics on THD of output voltages. We compare and analyze both approaches for their circuit configurations, voltage level generating method, THD characteristics of output voltage, efficiency, application areas, limitations, and other characteristics by experiments using $500[\mathrm{~W}]$ prototypes when they generate a 27-level output voltage.
\end{abstract}

Keywords: Efficiency, Inverters, Digital signal processor (DSP), Multilevel systems, Total harmonic distortion (THD), Transformers.

\section{Introduction}

A multilevel inverter can produce a high quality output voltage by synthesizing a large number of voltage levels similar to a sinusoidal wave. However, it needs to increase the number of components and has complexity problems for control and circuit configuration. To solve the problems, much research has focused on increasing the number of output voltages with a minimized number of components [1]-[10]. Among them, a presentable approach is to use the concept of the power of three when selecting the amplitude of dc voltage sources. To realize the effective concept, two kinds of approaches are reported in [4] and [5]. A common feature in both approaches is to use the concept of the power of three for selecting dc voltage sources, and a point of difference is whether it adopts a low frequency transformer or not, and where the transformer is located.

In [4], [10], it uses independent dc voltage sources prescaled in the power of three. The amplitude of the dc voltage sources is set to $V_{d c}, 3 V_{d c}, 3^{2} V_{d c}$ in sequence. Every independent dc voltage source is connected to the H-bridge and all terminals are connected in series. Therefore, a terminal voltage of H-bridge depends on the amplitude of

* Dept. of Electronics and Control Engineering, Hanbat National University, Korea. (feelsoon@ieee.org, feelsoon@hanbat.ac.kr swh1259@hanmail.net,k10216@nate.com,kskim@hanbat.ac.kr) Received 06 November 2012 Accepted 22 November 2012 the input dc voltage and an output voltage appears by the adding or subtracting of dc voltage sources connected in series. The most important issue of this approach is how to prepare independent dc voltage sources scaled in the power of three. When it takes three dc voltage sources scaled in the power of three, it will generate a 27 -level from $-13 V_{d c}$ to $+13 V_{d c}$ in an output voltage.

Another approach to realize the trinary concept is to use a cascaded transformer, which has secondary turn-ratios scaled in the power of three [5]-[7]. It needs a single dc voltage source $\left(V_{d c}\right)$ only. Other dc voltage sources $\left(3 V_{d c}\right.$ and $3^{2} V_{d c}$ ) are instantaneously generated by a cascaded transformer. Like that of the above mentioned approach, an output voltage is synthesized by addition or subtraction of instant dc voltage sources generated from each secondary of the transformer. When the transformer has three secondary terminals scaled in the power of three and connected in series, an output voltage will show a 27-level from $-13 V_{d c}$ to $+13 V_{d c}$ including a zero level.

Both approaches have several common features, at the same time, they show different characteristics, which limit application areas. To find the similarities and differences of both approaches, we compare them by experimental results based on 500 [W] prototypes when generating a 27-level output voltage.

2. Cascaded H-bridge Multilevel Inverters using 


\section{Voltage Sources Scaled in the Power of Three}

Generally, a cascaded H-bridge multilevel inverter includes an array of switching devices and independent $\mathrm{dc}$ voltage sources. The proper commutation of switches in $\mathrm{H}-$ bridges permits addition or subtraction of the dc voltages, which reach high voltage at the output, while the switching devices withstand only reduced voltages. Considering an array of individual dc voltage sources scaled in the power of three in sequence, continuous output voltage levels are synthesized by selection of suitable switching combinations. For example, we obtain continuous integers from -13 to +13 by using $\pm 1, \pm 3$, and \pm 9 .

\subsection{Comparison of Circuit Configurations}

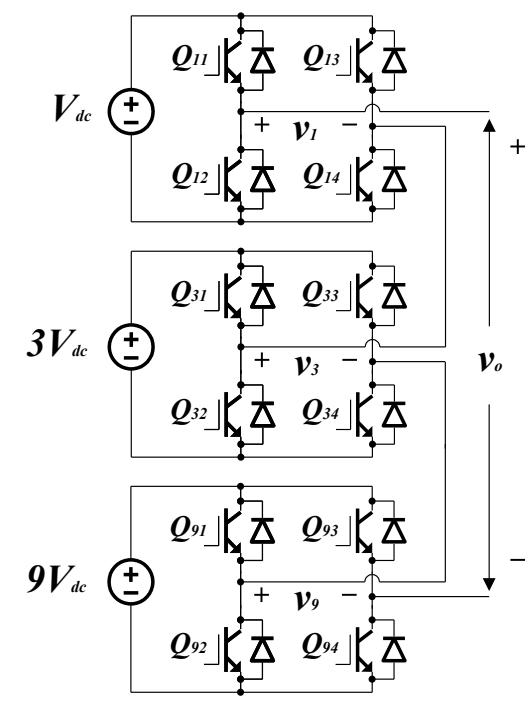

(a)

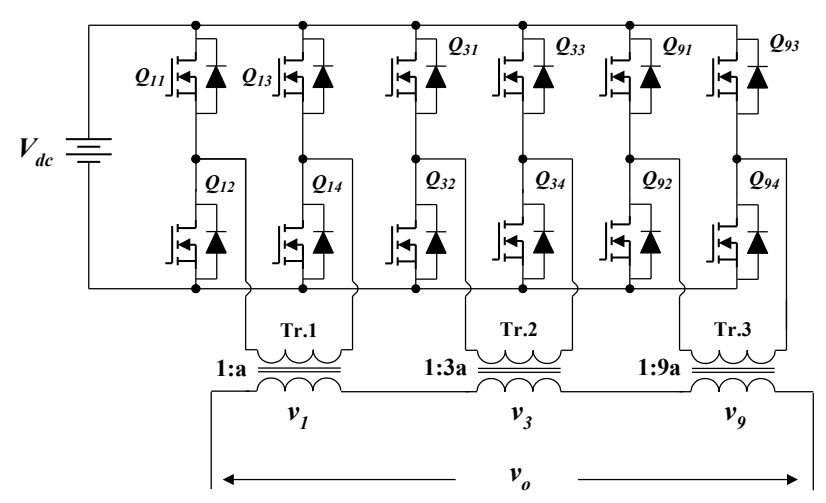

(b)

Fig. 1. Circuit configurations to realize trinary concept synthesizing a 27-level output voltage, (a) independent dc voltage sources scaled in the power of three in sequence, (b) instantaneous dc voltage sources scaled in the power of three by a cascaded transformer
Fig. 1(a) shows a circuit configuration of the first multilevel approach using a concept of the power of three [4]. In this circuit, the concept is applied to independent dc voltage sources. The circuit is similar to a traditional cascaded H-bridge multilevel inverter except the amplitude of independent dc voltage sources. To obtain independent dc voltage sources scaled in the power of three in sequence, battery stacks can be used and some front-end converters are required to maintain the amplitude of dc voltages constantly. A transformer with multi-winding outputs is usually adopted in practical applications.

Fig. 1(b) shows the second approach to realize the trinary concept using a cascaded transformer. It uses a single dc voltage source and a low frequency transformer having secondary turn-ratios scaled in the power of three. H-bridge cells are connected in parallel, and the secondary of the transformer is connected in series. It increases the volume and weight of the system because of the bulky size of the low frequency transformer.

Table 1. Comparison for the number of components

\begin{tabular}{lcc}
\hline No. of Components & Fig. 1(a) & Fig. 1(b) \\
\hline H-bridge Cell & 3 & 3 \\
Switch & 12 & 12 \\
DC Voltage Source & $\mathbf{3}$ & 1 \\
Transformer & 0 & $\mathbf{1}$ \\
\hline
\end{tabular}

Table 2. Comparison for voltage stress on switches

\begin{tabular}{ccc}
\hline Voltage Stress & Fig. 1(a) & Fig. 1(b) \\
\hline$Q_{11} \sim Q_{14}$ & $V_{d c}$ & $V_{d c}$ \\
$Q_{31} \sim Q_{34}$ & $3 V_{d c}$ & $V_{d c}$ \\
$Q_{91} \sim Q_{94}$ & $9 V_{d c}$ & $V_{d c}$ \\
\hline
\end{tabular}

Table 1 compares the number of components for both approaches. When generating an output voltage with a $27-$ level, both inverters need three H-bridge cells. Therefore, the number of switches in both is exactly same, but voltage stresses on switches are different according to the amplitude of dc voltage sources as shown in Table 2. As $V_{d c}$ increases, switches in Fig. 1(a) need a higher voltage rating, which results in the cost increasing. In the viewpoint of voltage stress, Fig. 1(b) is more useful than Fig. 1(a). It also means Fig. 1(b) is an effective method of circuit design and manufacturing because it uses switches which have the same voltage rating. However, Fig. 1(b) has a cascaded transformer which is required to synthesize instantaneous $\mathrm{dc}$ voltages scaled in the power of three. Because the transformer is operated in a low frequency, it is bulky and 
heavy.

\subsection{How to synthesize a multilevel output voltage wave in both approaches}

This section compares how to generate multilevel output voltage in both approaches. A basic idea is exactly the same because they use the same concept to generate a 27-level output voltage, i.e. using a combination of dc voltage sources scaled in the power of three. A main difference is how to realize the concept. Fig. 1(a) applies the concept to independent input dc voltage sources, and Fig. 1(b) applies to secondary turn-ratios of a cascaded transformer.

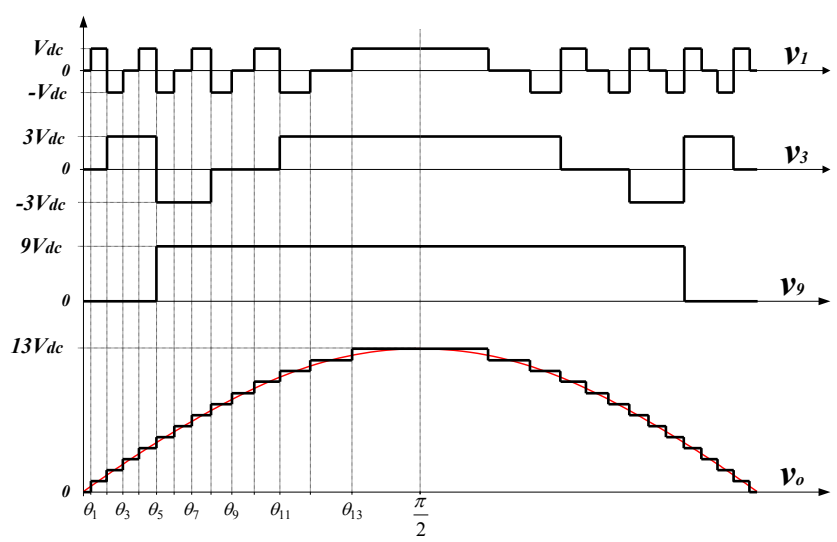

Fig. 2. Principle of synthesizing multilevel voltage

Fig. 2 shows a basic idea to generate 14 positive output voltage levels in both approaches. When it considers a negative wave, the output voltage has 27 levels. $v_{9}$ generates three levels in the output voltage wave, and then other voltages $\left(v_{3}\right.$ and $\left.v_{l}\right)$ add or subtract $V_{d c}$ or $3 V_{d c}$ levels to a fundamental wave to synthesize stepped waves. In an approach given in Fig. 1(a), the lowest H-bridge generates $v_{9}$ since it connects to $9 V_{d c}$. A middle H-bridge cell produces $3 V_{d c}$, and the upper one generates $V_{d c}$. Finally, three outputs connect in series to synthesize a final output voltage wave. On the other hand, H-bridge cells connected in parallel in Fig. 1(b) generate only $V_{d c}$ because all of them connect to a single dc voltage source $V_{d c}$. Scaling to the power of three is done by secondary turn-ratios of a cascaded transformer. A terminal having a turn-ratio of $1: 9 a$ generates $v_{9}$. It means $V_{d c}$ steps up $9 V_{d c}$ by means of the secondary winding. Other secondary terminals produce $3 V_{d c}$ and $V_{d c}$. Finally, three terminals connect in series to generate a final output voltage wave.

As shown in Fig. 2, waveforms of three terminal voltages are equivalent in both approaches, but the methodologies are different. Most of all, Fig. 1(a) uses constant voltage sources scaled in the power of three. On the other hand, Fig. 1(b) uses instantaneous voltages scaled by secondary turnratios having a sequence of the power of three.

In both approaches, the number of final output voltage levels is the sum of the three terminal voltages, and it is given as

$$
v_{o}=v_{1}+v_{3}+v_{9}=\sum_{k=0}^{\infty} v_{3^{k}}
$$

When $m$ number of H-bridge modules has dc voltage sources in sequence of the power of three, the number of output voltage level $(N)$ is defined by

$$
N=3^{m}, \quad m=1,2,3, \cdots
$$

Switching functions $\left(S_{F B k}\right)$ of an $\mathrm{H}$-bridge inverter are given by

$$
\begin{aligned}
& \text { if }\left(Q_{k 1}, Q_{k 4}\right)=\text { on then } S_{F B k}=1 \\
& \text { if }\left(Q_{k 1}, Q_{k 3}\right) \|\left(Q_{k 2}, Q_{k 4}\right)=\text { on then } S_{F B k}=0 \\
& \text { if }\left(Q_{k 2}, Q_{k 3}\right)=\text { on then } S_{F B k}=-1
\end{aligned}
$$

Here, $k=3^{n}$ and $n=0,1,2$. When it employs three $\mathrm{H}$ bridge modules, each terminal voltage of an H-bridge module is expressed by using switching functions.

$$
\begin{aligned}
& \text { if }\left(S_{F B 1}=1\right) \text { then } v_{1}=V_{d c} \\
& \text { if }\left(S_{F B 1}=0\right) \text { then } v_{1}=0 \\
& \text { if }\left(S_{F B 1}=-1\right) \text { then } v_{1}=-V_{d c} \\
& \text { if }\left(S_{F B 3}=1\right) \text { then } v_{2}=3 V_{d c} \\
& \text { if }\left(S_{F B 3}=0\right) \text { then } v_{2}=0 \\
& \text { if }\left(S_{F B 3}=-1\right) \text { then } v_{2}=-3 V_{d c} \\
& \text { if }\left(S_{F B 9}=1\right) \text { then } v_{3}=9 V_{d c} \\
& \text { if }\left(S_{F B 9}=0\right) \text { then } v_{3}=0 \\
& \text { if }\left(S_{F B 9}=-1\right) \text { then } v_{3}=-9 V_{d c}
\end{aligned}
$$

Finally, an output voltage is given by

$$
v_{o}=\sum_{k=0}^{\infty} 3^{k} \cdot V_{d c} \cdot S_{F B}
$$

Here, $S_{F B} \in\{1,0,-1\}$. In (7), an increase of $k$ means an increase of the number of dc voltage sources scaled in the power of three. When it has three dc voltage sources, an output voltage is expressed as

$$
v_{o}=\left(S_{F B 1}+3 S_{F B 3}+9 S_{F B 9}\right) \cdot V_{d c}
$$

Table 3 lists switching functions required to synthesize a positive output voltage in both approaches. For a negative voltage wave, it can be easily obtained by multiplying -1 to 
Table 3. Here, $S_{F B 9}$ is a switching function of an inverter generating $9 V_{d c}$. It takes a naught when the desired output level is equivalent to or lower than the fourth level in Table 3. When a desired output voltage level is higher than the fourth level, it requires unity. $S_{F B 9}$ can be expressed by using C-language as (9). Here, all variables are integers (int). $m$ means the number of output voltage levels.

$$
\begin{aligned}
& \text { if }(m \leq 4) \text { then } S_{F B 9}=0 \\
& \text { if }(m>4) \text { then } S_{F B 9}=1
\end{aligned}
$$

Switching function $S_{F B 3}$ of an inverter generating $3 V_{d c}$ is determined by

$$
\begin{aligned}
& \text { if }\left(\frac{m+1}{3}\right) \% 3=0 \text { then } S_{F B 3}=0 \\
& \text { if }\left(\frac{m+1}{3}\right) \% 3=1 \text { then } S_{F B 3}=1 \\
& \text { if }\left(\frac{m+1}{3}\right) \% 3=2 \text { then } S_{F B 3}=-1
\end{aligned}
$$

where $\%$ is a modulus operator. Finally, switching function $S_{F B I}$ of an inverter generating $V_{d c}$ is determined by

$$
\begin{aligned}
& \text { if }(m \% 3)=0 \text { then } S_{F B 1}=0 \\
& \text { if }(m \% 3)=1 \text { then } \quad S_{F B 1}=1 \\
& \text { if }(m \% 3)=2 \quad \text { then } \quad S_{F B 1}=-1
\end{aligned}
$$

Table 3. Switching function for generating a zero and 13 positive voltage levels.

\begin{tabular}{cccc}
\hline $\begin{array}{c}\text { Output Voltage Level } \\
(\mathrm{m})\end{array}$ & $S_{F B 9}$ & $S_{F B 3}$ & $S_{F B 1}$ \\
\hline 0 & 0 & 0 & 0 \\
1 & 0 & 0 & 1 \\
2 & 0 & 1 & -1 \\
3 & 0 & 1 & 0 \\
4 & 0 & 1 & 1 \\
5 & 1 & -1 & -1 \\
6 & 1 & -1 & 0 \\
7 & 1 & -1 & 1 \\
8 & 1 & 0 & -1 \\
9 & 1 & 0 & 0 \\
10 & 1 & 0 & 1 \\
11 & 1 & 1 & -1 \\
12 & 1 & 1 & 0 \\
13 & 1 & 1 & 1 \\
\hline
\end{tabular}

\subsection{Comparison of Total Harmonic Distortion}

In both multilevel inverters, when applying Fourier series analysis to an output voltage, the amplitude of $n$-th harmonic component is determined by

$$
V_{n}=\frac{4 V_{d c}}{n \pi} \sum_{k=1}^{s} \cos \left(n \theta_{k}\right)
$$

where $n$ is an odd harmonic order. Amplitudes of all even harmonics are zero. $s$ means the number of the output voltage level; here, it becomes 13. $\theta_{k}$ is the switching angles that indicate on or off instants of switches inside H-bridge modules. Modulation index $(M)$ is defined as

$$
M=\frac{\pi}{4} \cdot \frac{V_{1}}{V_{\max }}=\frac{\pi}{4} \cdot \frac{V_{1}}{s \cdot V_{d c}}
$$

Here, $V_{l}$ is a fundamental component of an output voltage. $V_{\max }$ is the maximum attainable output voltage of an inverter. When it generates 27 levels in the output voltage, $s$ becomes 13 and $V_{\max }$ is $13 V_{d c}$.

As given in (12), both inverters have basically the same total harmonic distortions in output voltage. However, THD of Fig. 1(b) is affected by a load condition. A leakage inductance of a cascaded transformer acts as a high performance low-pass filter when increasing an output current. As a result, voltage levels collapse, and a stepped output voltage wave changes into a sinusoidal wave at a heavy load condition. It means Fig. 1(b) shows an improved THD characteristic when the output load increases.

\section{Experimental Result and Discussion}

For a practical comparison, we carry out experiments on both inverters. Two prototypes of 500 [W] are designed and controlled by a digital controller using a DSP28335. Specifications of prototypes are listed in Table 4. In practical applications, it is somewhat difficult to find

\begin{tabular}{|c|c|c|c|}
\hline Component & Specification & \multicolumn{2}{|c|}{ Value } \\
\hline \multirow{3}{*}{ Switch } & & $V_{d s s}$ & $400 \mathrm{~V}$ \\
\hline & IRFP360 & $\boldsymbol{R}_{d s(o n)}$ & $0.2 \mathrm{ohm}$ \\
\hline & & $I_{d}$ & $25 \mathrm{~A}$ \\
\hline Controller & $D S P$ & \multicolumn{2}{|c|}{ TMS320F28335 } \\
\hline \multirow{3}{*}{$\begin{array}{c}\text { Input DC } \\
\text { Voltage Source }\end{array}$} & Battery & $12 \mathrm{~V}$ & $25 \mathrm{AH}$ \\
\hline & Fig. 1(a) & $\begin{array}{l}V_{d c} \\
3 V_{d c} \\
9 V_{d}\end{array}$ & $\begin{array}{c}\text { Series } \\
\text { Connection }\end{array}$ \\
\hline & Fig. 1(b) & \multicolumn{2}{|c|}{$V_{d c}$} \\
\hline \multirow{2}{*}{$\begin{array}{c}\text { Cascaded } \\
\text { Transformer }\end{array}$} & Fig. 1(a) & \multicolumn{2}{|c|}{0} \\
\hline & Fig. 1(b) & \multicolumn{2}{|c|}{ 1:3:9 (Secondary) } \\
\hline \multirow{2}{*}{ Output Voltage } & \multirow{2}{*}{$\begin{array}{c}\text { 27-level } \\
\text { including zero }\end{array}$} & $V_{o}$ & $110 V_{a c}$ \\
\hline & & $f$ & $60 \mathrm{~Hz}$ \\
\hline
\end{tabular}
independent dc voltage sources. Although a battery can be used for independent voltage source, it needs a front-end converter to maintain dc voltage constantly. To make three dc voltage sources scaled in the power of three in Fig. 1(a), three and nine batteries are connected in series and maintain constant voltages by each front-end converter. In a case of Fig. 1(b), 13 batteries are connected in parallel to supply sufficient power to an output load.

Table 4. Specifications of prototypes. 


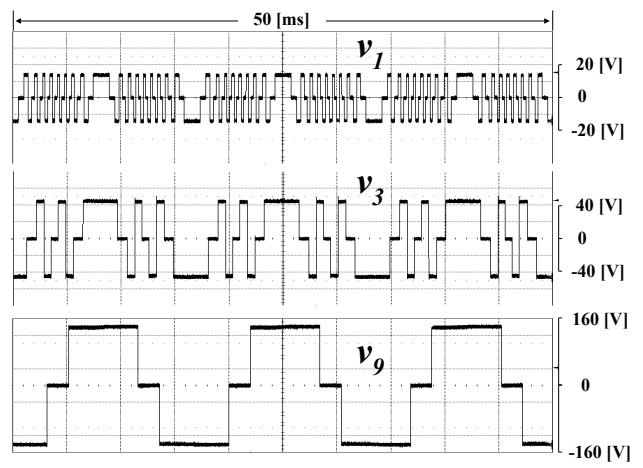

(a)

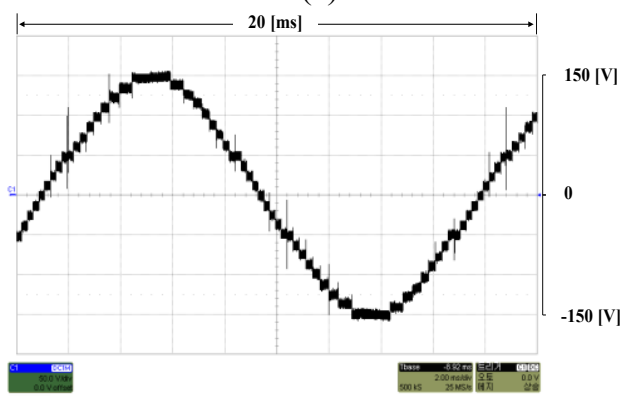

(b)

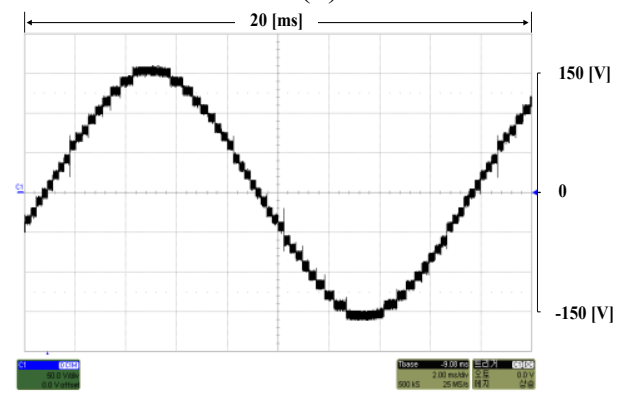

(c)

Fig. 3. Experimental results for a multilevel inverter employing independent dc voltage sources scaled in the power of three given in Fig. 1(a), (a) output terminal voltages of each inverter $v_{l}, v_{3}$, and $v_{9},(\mathrm{~b})$ output voltage at a no load, $v_{o}$, (c) output voltage with a resistive load of $20[\Omega]$

Fig. 3 shows experimental results for a multilevel inverter given in Fig. 1(a). It employs three independent dc voltage sources scaled in the power of three. Fig. 3(a) shows three terminal voltages to synthesize a 27-level output voltage. An inverter with a $9 V_{d c}$ voltage source generates three basic levels $\left(v_{9}\right)$ with amplitude of $\pm 9 V_{d c}$. An inverter with a $3 V_{d c}$ voltage source adds or subtracts $\pm 3 V_{d c}$ level to the fundamental voltage level. Finally an inverter with a $V_{d c}$ voltage source adds or subtracts $\pm V_{d c}$ level to the basic voltage level. Therefore, it can synthesize 27 levels from $+13 V_{d c}$ to $-13 V_{d c}$ including a zero level. Fig. 3(b) and Fig. 3 (c) show output voltages at a no load and with a resistive load of $20[\Omega]$, respectively. In both load conditions, output voltages are similar to sinusoidal one owing to 27 levels.

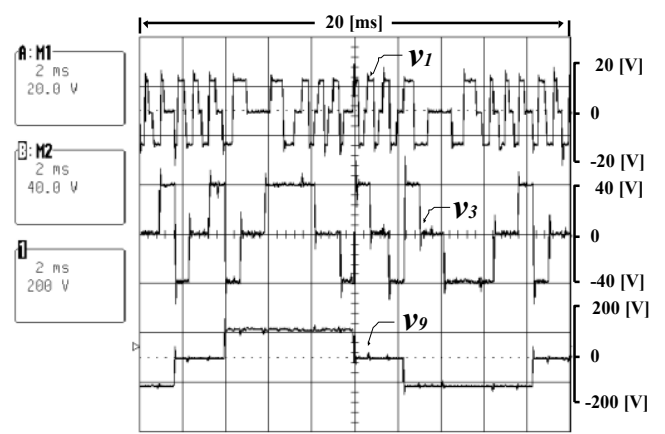

(a)

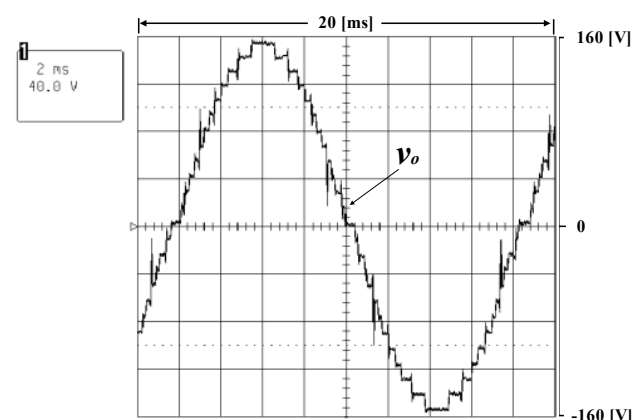

(b)

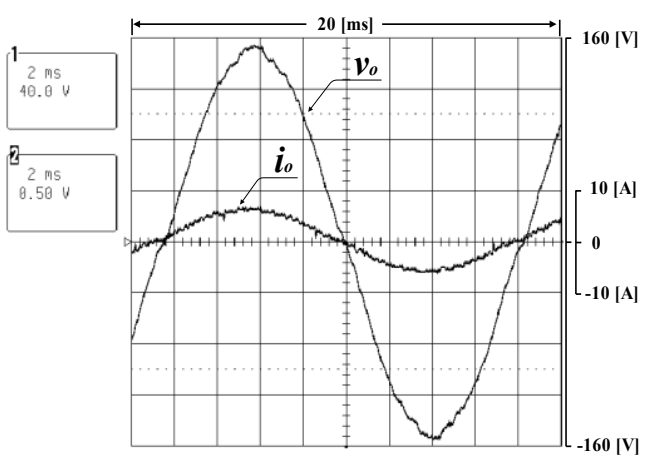

(c)

Fig. 4. Experimental results for a cascaded transformer based multilevel inverter given in Fig. 1(b), (a) output terminal voltages of each inverter $v_{l}, v_{3}$, and $v_{9}$, (b) output voltage at a no load, $v_{o}$, (c) output voltage and current with a resistive load of $20[\Omega]$

Fig. 4 shows experimental results of a multilevel inverter employing a cascaded transformer given in Fig. 1(b). Fig. 4(a) shows each secondary voltage. As shown in Fig. 4(b), it has a 27-level in an output voltage by combination of $v_{l}$, $v_{3}$, and $v_{9}$. It shows critical 27 levels in an output voltage at a no load. When a load current increases, an output voltage turns into a more sinusoidal wave since a leakage inductance of a cascaded transformer operates as a highperformance filter as shown in Fig. 4(c). At a no load, output voltages of both inverters have some harmonic components such as 3, 5, 7, 11, and other low order harmonics, but they are sufficiently eliminated as shown in Fig. 4(c) owing to the filtering effect of reactance of a 
cascaded transformer. From these facts, it is clear that a cascaded transformer based multilevel inverter in higher load conditions can produce more sinusoidal output voltage than a multilevel inverter employing independent $\mathrm{dc}$ voltage sources scaled in the power of three.

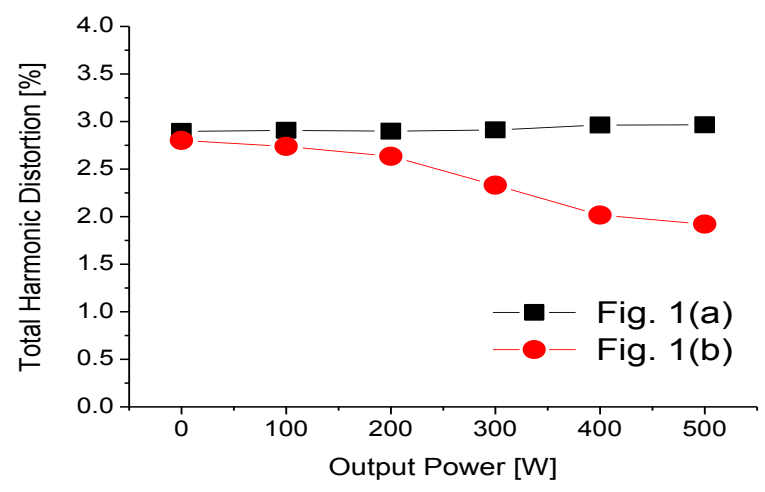

Fig. 5. THD of output voltage in both approaches

Fig. 5 shows THD comparison for both approaches. When output power increases, an approach given in Fig. 1(a) does not change THD regardless of output power, and it maintains below 3[\%]. On the other hand, an approach given in Fig. 1(b) shows a similar THD at a no load and low loaded, but it changes THD when increasing output power. It shows about $1.9[\%]$ at $500[\mathrm{~W}]$ loaded due to the filtering effect of the leakage inductance.

Table 5 lists a component comparison between a cascaded H-bridge multilevel inverter and both inverters when generating 27 output levels. In the viewpoint of generating a large number of output voltage levels, a conventional cascaded H-bridge multilevel inverter is not suitable because of a large number of switching devices. From the comparison, it is clear that the most outstanding advantage of both approaches is the reduction of the number of switches. It can save $10 \mathrm{H}$-bridge modules resulting in a saving of 40 switches.

Each cell of the cascaded type requires its own isolated power supply. The provision of these isolated supplies is the main limitation in the power electronic circuit design. Above all, amplitude of the input dc voltage sources in a multilevel inverter given in Fig. 1(a) is as different as the power of three. In practical application, series connected batteries can be used for the power sources scaled in the power of three. However, to produce $9 V_{d c}$, it needs nine batteries maybe connected in series. It decreases structural stability and reliability. If it receives the input dc source from an ac grid, it needs a transformer to supply different voltages as given in Table 5. However, as the low frequency transformer is located at the front-end of the inverter, it does not have any influence on the operating frequency of the output voltage. It means that it can be applied to motor drives using a VF control because it is free from the saturation of the transformers. However, because of the voltage rating of the H-bridge cell connected to $9 V_{d c}$, it is difficult to apply to a high voltage application. This method can be used as an alternative for PWM inverters to decrease a switching noise and loss.

The cascaded transformer based inverter given in Fig. 1(b) needs low frequency transformers, which have turn-ratios scaled in the power of three. It increases the size of the system and volume. It will decrease efficiency due to the transformer in itself. In addition, this method is not desirable for motor drives employing a VF control method because of the transformer saturation. Therefore it is suitable for CVCF (constant voltage and constant frequency) applications such as UPS (uninterruptible power supply), photovoltaic power generating inverter systems.

Table 5. Components comparison with a conventional cascaded H-bridge multilevel inverter

\begin{tabular}{cccc}
\hline & \multicolumn{3}{c}{ The number of Components } \\
\cline { 2 - 4 } Items & $\begin{array}{c}\text { Cascaded } \\
\text { H-bridge Cell }\end{array}$ & Fig. 1(a) & Fig. 1(b) \\
\hline $\begin{array}{c}\text { H-bridge Module } \\
\text { Switch }\end{array}$ & 13 & 3 & 3 \\
$\begin{array}{c}\text { Independent } \\
\text { CC Voltage Source }\end{array}$ & 52 & 12 & 12 \\
$\begin{array}{c}\text { Transformer } \\
\text { (Input) }\end{array}$ & 13 & 3 & 1 \\
$\begin{array}{c}\text { Transformer } \\
\text { (Output) }\end{array}$ & 13 & $V_{d c} 3 V_{d c}{ }^{9} V_{d c}$ & Single-winding \\
& 0 & 0 & 1 \\
\hline
\end{tabular}

\section{Conclusion}

This paper compared two 27-level inverters using the concept of the power of three to dc voltage sources. A common feature in both approaches is to use the concept of the power of three for dc voltage sources, and a point of difference is whether it adopts a low frequency transformer or not, and where the transformer is located. According to the differences, application areas are limited and show different characteristics on THD of output voltages.

By experiments using 500 [W] prototypes, we compared and analyzed both approaches for circuit configurations, the voltage level generating method, THD characteristics of the output voltage, efficiency, application areas, limitations, and other characteristics.

\section{References}

[1] L. G. Franquelo, J. Rodriguez, S. Kouro, R. Portillo, and M. A. M. Prats, "The age of multilevel converter arrives," IEEE Ind. Electron. Magazine, pp. 28-39, 2008.

[2] J. Rodriguez, J. S. Lai, and F. Z. Peng, "Multilevel Inverters: A survey of topologies, controls, and applications," IEEE Trans. Ind. Electron., vol. 49, no. 4, pp. 724-738, Aug. 2002. 
[3] J. S. Lai, and F. Z. Peng, "Multilevel Converters-A New Breed of Power Converters," IEEE Trans. Ind. Appl., vol. 32, no. 3, pp. 509-517, May/June, 1996.

[4] C. S. Kwon, W. K. Choi, U. T. Hong, S. H. Hyun, and F. S. Kang, "Cascaded H-bridge Multilevel Inverter Using Trinary DC Sources," Proc. of ICEMS, Oct. 10-13, 2010, pp. 52-55.

[5] F. S. Kang, S. J. Park, M. H. Lee, and C. U. Kim, "An efficient multilevel synthesis approach and its application to a 27-level inverter," IEEE Trans. Ind. Electron., vol. 52, no. 6, pp. 1600-1606, Dec. 2005.

[6] F. S. Kang, S. J. Park, C. U. Kim, and T. Ise, "Multilevel PWM inverters suitable for the use of stand-alone photovoltaic power system," IEEE Trans. Energy Conv., vol. 20, no. 4, pp. 906-915, Dec. 2005.

[7] F. S. Kang, "A modified cascade-transformer-based multilevel inverter and its efficient switching function," Electric Power Systems Research, vol. 79, pp. 1648-1654, 2009.

[8] S. G. Song, F. S. Kang, and S. J. Park, "Cascaded multilevel inverter employing three-phase transformers and single $\mathrm{dc}$ input," IEEE Trans. Ind. Electron., vol. 56, no. 6, pp. 20052014, June 2009.

[9] Y. Liu, and F. L. Luo, "Trinary hybrid 81-level multilevel inverter for motor drive with zero common-mode voltage," IEEE Trans. Ind. Electron., vol. 55, no. 3, pp. 1014-1021, 2008.

[10] Y. Liu, and F. L. Luo, "Trinary hybrid multilevel inverter used in STATCOM with unbalanced voltages," IEE Proc. on Electric Power Appl., vol. 152, no. 5, pp. 1203-1222, 2005.

\section{Acknowledgement}

This research was supported by 2010 academic research program funded by Hanbat National University

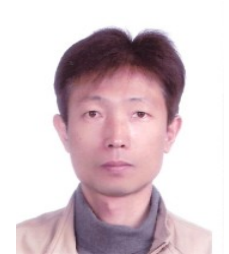

Seok-Hwan Hyun received B.S. and M.S degrees in the Department of Electronics and Control Engineering at Hanbat National University, Daejeon, Korea in 2006 and 2011, respectively. From 2010, he is a president of S\&D system. His research interests are in the area of IT based power electronics.

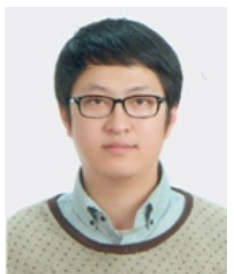

Cheol-soon Kwon received B.S. and M.S. degrees in the Department of Electronics and Control Engineering at Hanbat National University, Daejeon, Korea in 2010 and 2012, respectively. From 2012, he is with a PNE solution company as an engineer. His research interests are in the area of power electronics including design and control of power converters for electric vehicles. Mr. Kwon is a member of KIEE and KIPE.

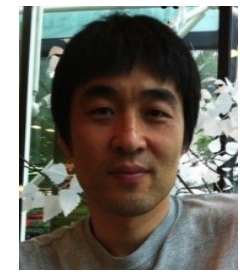

Kwang-soo Kim received the BS, MS, and $\mathrm{PhD}$ degree in electrical and computer engineering, all from the Seoul National University in 1996, 1998, and 2004, respectively. He worked for Samsung Electronics from January 2004 to March 2007 and Hyundai Motor Company from April 2007 to February 2008. Since March 2008, he has been an Assistant Professor in the Department of Electronics and Control Engineering at Hanbat National University. His research interests include system modeling, sensor fusion, and control system design and analysis.

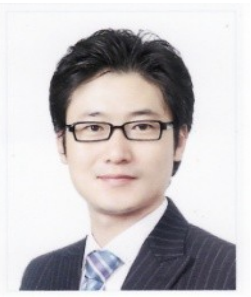

Feel-soon Kang received M.S. and Ph.D. degrees from Pusan National University, Busan, Korea in 2000 and 2003, respectively. From 2003 to 2004, he was with the Department of Electrical Engineering, Osaka University, Osaka, Japan as a Postdoctoral Fellow. Since 2004, he has been with the Department of Electronics and Control Engineering, Hanbat National University, Daejeon, Korea as an associate professor. From 2012 to 2013, he is a visiting professor in the Department of Electrical and Computer Engineering, Colorado State University. His research activities are in the area of power electronics including design and control of power converters for electric vehicles, and multilevel inverters for photovoltaic power generating systems. He received an Award from IEEE Industrial Electronics Society and the Best Presentation Prize at IEEE IECON'01 be held in Denver, Colorado USA in 2001. He was honored an Academic Award from Graduate School of Pusan National University and Habat National University in 2003 and 2005, respectively. And he also received several Best Paper Awards from The Korean Institute of Power Electronics (KIPE), The Korean Institute of Electrical Engineers (KIEE), and The Korea Institute of Maritime Information and Communication Science (KIMICS). He served as Cochairs and secretary for ICEMS 2010, MAGLEV 2011, VPPC 2012, and other domestic and international conferences. Dr. Kang is an Associate Editor in the IEEE Transactions on Industrial Electronics and JICEMS. 